\title{
DYNAMIC GARMENT SIMULATION BASED ON HYBRID BOUNDING VOLUME HIERARCHY
}

\author{
Dongyong Zhu, Zhong Li*, Feng Xia, Yong Xu
}

\author{
Dongyong Zhu, Zhong Li* Feng Xia, Yong Xu \\ College of Science, Zhejiang Sci-Tech University, Hangzhou, China, 310018 \\ *Corresponding author. Email: lizhong@zstu.edu.cn
}

\section{Abstract:}

In order to solve the computing speed and efficiency problem of existing dynamic clothing simulation, this paper presents a dynamic garment simulation based on a hybrid bounding volume hierarchy. It firstly uses MCASG graph theory to do the primary segmentation for a given three-dimensional human body model. And then it applies K-means cluster to do the secondary segmentation to collect the human body's upper arms, lower arms, upper legs, lower legs, trunk, hip and woman's chest as the elementary units of dynamic clothing simulation. According to different shapes of these elementary units, it chooses the closest and most efficient hybrid bounding box to specify these units, such as cylinder bounding box and elliptic cylinder bounding box. During the process of constructing these bounding boxes, it uses the least squares method and slices of the human body to get the related parameters. This approach makes it possible to use the least amount of bounding boxes to create close collision detection regions for the appearance of the human body. A spring- mass model based on a triangular mesh of the clothing model is finally constructed for dynamic simulation. The simulation result shows the feasibility and superiority of the method described.

\section{Keywords:}

Garment simulation; Hybrid bounding box; Collision detection

\section{Introduction}

Cloth simulation based on the physical model is an important research topic and remains a challenging issue of Garment Computer Aided Design (GCAD) in recent years. Cloth simulation requires multiple skills knowledge in fields covering garment engineering, computer graphics, mathematics and physics. These skills are vital in focusing on generating the dynamic results of movement and deformation of cloth in such a manner as to appear authentic, fast and realistic. It has been widely used in virtual dressing, character simulations, movies, games, and similar circumstances.

During the process of dynamic clothing simulation, the challenges mainly relate to the construction of an effective physical model of clothing and the collision detection between the human body and cloth. The first problem for cloth simulation is the establishment of a physical model. Traditional clothing models comprise of energy-based particle systems ${ }^{[1]}$ and mass- spring models ${ }^{[2-4]}$. Because mass-spring models are easy to construct and can quickly simulate elasticity, flexibility of cloth in a realistic manner, this method is most widely used in GCAD. Another problem of cloth simulation is the numerical solution of the dynamics of differential equations. Explicit time integration methods ${ }^{[5-7]}$ have low computational complexity when dealing with differential equations that have a large rigidity coefficient. But the overall efficiency of the method is poor because in order to ensure stability, explicit time integration methods only use a small integration step. Implicit time integration methods ${ }^{[8,9]}$ are unconditionally stable because they can use bigger integration steps. However, they also need to solve the equations and are related to more approximations, which result in over- damping. These factors seriously affect the quality of the simulation. In order to combine the advantage of explicit methods and implicit methods, some scholars have also suggested semi-implicit methods ${ }^{[10,11]}$ and mixed integral methods ${ }^{[12,13]}$. In the actual development of the cloth simulation, the most critical issue is how to efficiently deal with the collisions between cloth and the body in real time. The challenge that needs to be solved is how to reduce the number of patches that are handled in the collision and the time taken for collision detection. Bounding box technology can use relatively simple geometric objects to simulate complex objects approximately and realize the goal of reducing the processing time for collisions. Bounding box technology is widely used and includes methods such as sphere bounding box, axisaligned bounding box (AABB), oriented bounding box (OBB) ${ }^{[14]}$, k-DOPs and other variations. Feng et al. ${ }^{[15]}$ constructed a bounding capsule for every bone cluster and build a sphere set approximation for the body mesh and assign each sphere to a corresponding bone cluster. Unfortunately, the sphere is isotropic and it is easy to create a larger error due to the representation of the sharp and flat areas. This problem can be weakened by selecting an ellipsoid as the bounding volume. Bischoff et al. ${ }^{[16]}$ applied initial seed points to construct an ellipsoid for each decomposed model, but it cannot simplify the complexity of the computing time when only using the ellipsoid approximation. Bergen et al. ${ }^{[17]}$ introduced an axis-aligned bounding box for collision detection. Hutter and Fuhrmann ${ }^{\left[{ }^{[8]}\right.}$ constructed k-DOPs to improve the efficiency of collision detection. Chang et al. ${ }^{[19]}$ used an oriented bounding box to reduce the time of collision detection. 
In this paper, the authors present a method for dynamic garment simulation based on a hybrid bounding volume hierarchy. The method collects the basic collision units of a human body model by using MCASG graph theory ${ }^{[20]}$ and K-means clustering algorithm [21]. Then it constructs cylinder bounding box, elliptical cylinder bounding box and sphere box to approximate these basic units. It can improve the efficiency of collision detection to realize fast and realistic clothing simulation.

\section{The process of cloth simulation base on hybrid bounding volume hierarchy}

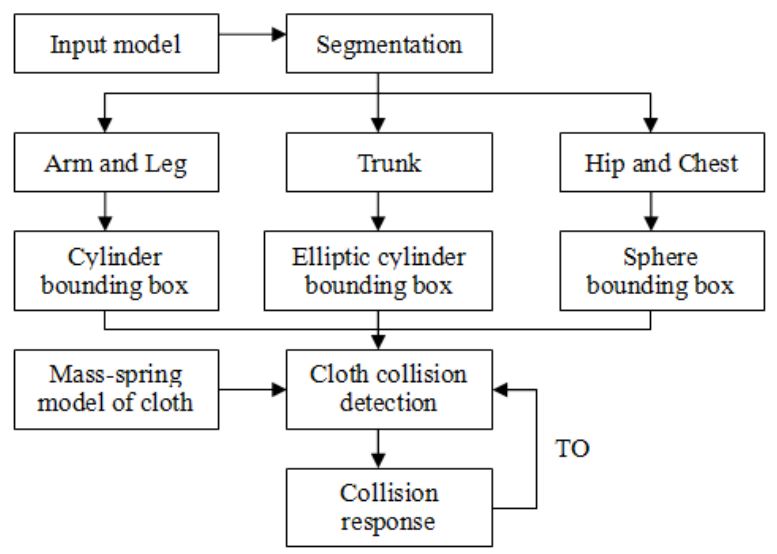

Figure 1: Main process of cloth simulation

This cloth simulation firstly divides a three-dimensional human body model into separate parts by the initial segmentation. It then does the secondary segmentation to obtain the collision units such as upper and lower arms, upper and lower legs, trunk, hip, women's chest, etc. Correspondingly, it constructs different kinds of bounding boxes such as cylinder bounding box, elliptical cylinder bounding box and sphere bounding box to approximate these units. Finally it realizes the cloth simulation according to the fast collision detection and response for these bounding boxes. The main process is illustrated in Figure 1.

\section{Segmentation of three-dimensional human model}

\subsection{Initial segmentation of human model based on MCASG graph}

We use MCASG graph algorithm [20] to process initial segmentation. The geometric aspect of an object is described as curvedness in the segmentation, which is also known as the bending energy. It measures the intensity of the surface curvature and describes how gently or strongly curved a surface is. Mathematically, it is defined as

$$
C_{v}=\sqrt{\left(k_{\max }^{2}(v)+k_{\min }^{2}(v) / 2\right.},
$$

where $k_{\max }(v)$ and $k_{\min }(v)$ are the principal curvatures at vertex $v$ on a surface and can be computed by mean curvature $H(v)$ and Gaussian curvature $K(v)$

$$
\left\{\begin{array}{l}
k_{\max }(v)=H(v)+\sqrt{H^{2}(v)-K(v)} \\
k_{\min }(v)=H(v)-\sqrt{H^{2}(v)-K(v)} .
\end{array}\right.
$$

Detailed estimation of mean curvature and Gaussian curvature of each vertex on a three-dimensional mesh model can be gotten from literature $[22,23]$.

In the initial segmentation, an input mesh is defined as an attributed graph $G[(V, C), E]$, where $V(G)=\left\{v_{1}, v_{2}, \ldots, v n\right\}$ is the set of vertices comprising the mesh, $C(G)=\left\{C_{v 1}, C_{\mathrm{v} 2}, \ldots, C v n\right\}$ is the set of curvedness values associated with the vertices in the mesh, and $E(G)$ is a set of edges connecting the vertices in $V(G)$. Vertices $v i$ and $v j$ are adjacent and are neighbors if there exists an edge eij connecting them. The neighborhood $N(v i)$ of a vertex vi consists of a set of vertices that are adjacent to vertex vi.

Given an input mesh $G$ and a curvedness threshold range [ $t k$, $\left.t k_{+1}\right)$, the process of segmentation is described as follows:

\section{(1) Initialization step}

Select an arbitrary vertex $v$ from $G$ such that its curvedness value satisfies the threshold range. Set $V\left(M_{0}\right)=v, E\left(M_{0}\right)=\phi$, $C\left(M_{0}\right)=C v$.

\section{(2) Iteration step}

a) Dilated graph extraction. Determine the neighbors of the vertices in $V\left(M k^{-1}\right)$ and perform median filtering on their curvedness values. Extract the dilated graph $G_{d}^{k}$ such that

$$
\begin{aligned}
& V\left(G_{d}^{k}\right)=\left\{V\left(M^{k-1}\right) \cup v^{\prime} \mid v^{\prime} \in N(v), \exists v \in V\left(M^{k-1}\right)\right\}, \\
& C\left(G_{d}^{k}\right)=\left\{C_{v}^{\prime}\right\}, \text { where } \forall v \in V\left(G_{d}^{k}\right) \quad C_{v}^{\prime}=\left\{\begin{array}{l}
\operatorname{median}\left\{C_{v^{\prime}} \mid v^{\prime} \in N(v)\right\}, C_{v} \notin\left[t_{i}, t_{i+1}\right) \\
C(v), \\
\text { otherwise }
\end{array}\right. \\
& E\left(G_{d}^{k}\right)=\left\{e_{u v} \in E(G) \mid u, v \in V\left(G_{d}^{k}\right)\right\} .
\end{aligned}
$$

b) The removal of the outlier vertices in the dilated graph will result in a graph $M k$ where

1) $V\left(M^{k}\right)=\left\{v \in V\left(G_{d}^{k}\right) \mid C_{v} \in\left[\mathrm{t}_{i}, \mathrm{t}_{i+1}\right)\right\}$,

2) $E\left(M^{k}\right)=\left(V\left(M^{k}\right) \times V\left(M^{k}\right)\right) \cap E\left(\mathrm{G}_{d}^{k}\right)$,

3) $C\left(M^{k}\right)=\left\{C_{v}, \forall v \in V\left(M^{k}\right)\right\}$.

All the vertices in $M k$ will have their curvedness values in the desired curvedness threshold interval by performing above steps. When the iteration process converges namely $M k=M$ $k^{-1}$, these vertices are regarded in the same region.

Therefore, different regions of the human model can be segmented by MCASG algorithm. The initial segmentation result of a human body model is illustrated in Figure 2.1. 


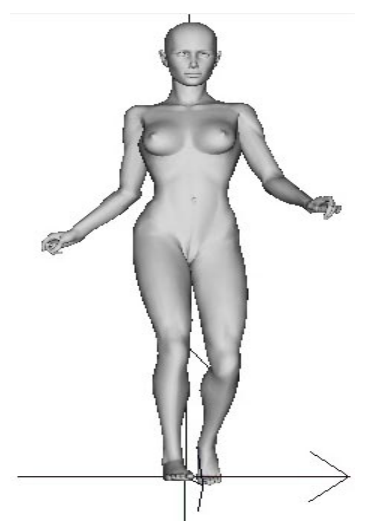

a) The original human model Figure 2.1. Initial segmentation

\subsection{The second segmentation based on K-means clustering algorithm}

The initial result of segmentation is not enough to establish the bounding box for the fast collision detection. Therefore a second segmentation is required. The K-means clustering algorithm ${ }^{[21]}$ is traditionally used to classify the position of each data point on the prototype through the use of re-iterative objective functions. Here, we apply the K-means clustering algorithm to process the second segmentation of the human body model.

\section{(1) The second segmentation of the leg}

The curvature of each vertex on the leg of the human body model is firstly analyzed. $S$ is the set of vertices whose curvature exceeds a threshold value and K-means clustering is then carried out for $S$. $K$ is set to 3 and the initial centers are set to three clusters for the highest point, the lowest point, and the nearest point from the mean position of the highest and the lowest points.

Points of set $S$ are divided into corresponding clusters in turn and the group of clusters which correspond with the upper leg, knee and the lower leg are obtained. Three sets of cluster points are set as $A, B, C$ respectively and it uses the mean point of the cluster points of the knee to segment the upper leg and lower leg.

We further segment the feet and shank regions from the lower leg. First, the direction that is from the center of set $C$ to the center of set $B$ is calculated as the direction vector $n$. Then the point which is the nearest from the center of set $B$ in set $C$

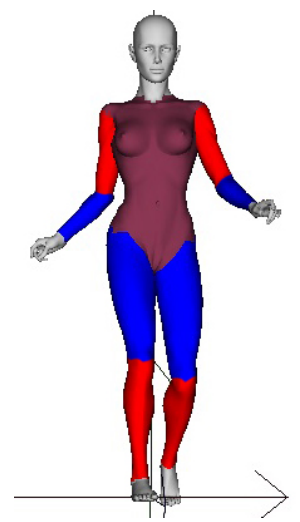

Figure 2.2. The second segmentation

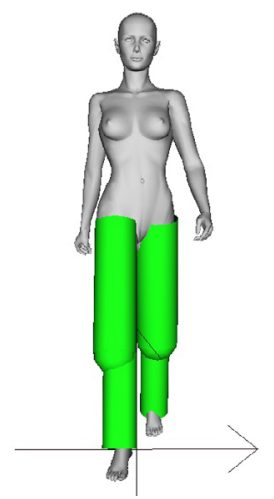

Figure 3.1. Leg bounding box is selected. A plane is constructed by including this point and setting its normal vector as $n$. The plane is used to cut the lower leg to separate the feet from the shank. Therefore, it segments the upper leg, knee, shank and feet parts from the original leg.

\section{(2) The segmentation for the upper arm and forearm}

The segmented method is similar to the above method. The result of segmentation is illustrated in Figure 2.2.

\section{Hybrid bounding volume and collision detection}

\subsection{Hybrid bounding volume of human model}

The closest fitting and efficient hybrid bounding box is chosen for local human body regions in dynamic clothing simulation. Here, legs and arms are approximated by a cylinder bounding box, the trunk is replaced by an elliptic cylinder bounding box, the hip and woman's chest is set as a sphere bounding box. By doing so, these approximations can effectively improve the computational efficiency of the collision detection. For the human body model, we firstly construct the oriented bounding box (the longest edge is set as the girth, then the face direction (the front direction of the body) is judged according to the rule that the curvature variance of the segmented face part is more obvious than the back part of the head.

\subsubsection{Leg and arm}

The leg is approximated by a cylinder bounding box. The computation of a cylinder bounding box construction is about double that of a sphere bounding box, but the close degree that a cylinder bounding box fits to the leg is higher than the degree that multiple sphere bounding boxes would fit to it. For the cylinder box approximating the arm and leg part of the human body model, we construct the oriented bounding box (the longest edge is set as the height of the cylinder box), and then use the slice intersection process to determine the base circle with a given radius. First, a slice on the upper and lower part of the leg respectively is carried out. Suppose $P_{0}$ is defined as the center point of a loop of the slice, and $x i(i=1, \ldots, n)$ are the measuring points on this loop. It defines $R=\max \left\{x i P_{0} i=1, \ldots, n\right\}$ as the radius of the circle to create the cylinder bounding box with the height information of the leg. The result is illustrated in Figure 3.1 .

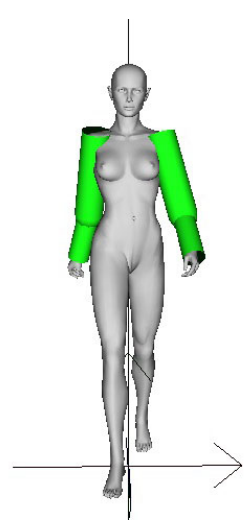

Figure 3.2. Arm bounding box 
Because the arm is also replaced by a cylinder bounding box, the approximation process is the same as in the leg. The result is illustrated in Figure 3.2.

\subsubsection{Woman's chest}

In approximating a woman's chest, a sphere bounding box is used in the simulation. We also apply the slice intersection process to determine the height of the center of sphere, then use the least squares method to determine the center and radius of the sphere. A slice of the woman's chest is taken horizontally, and the height of the slice is determined by the outermost point of the torso in a direction toward that of the human faces. This produces a number of measuring points on two semicircles.

For one semicircle, there are $n$ measuring points, which are illustrated in Fig3.3. We get an equation of the straight line over any two points, such as $P_{i}$ and $P_{j}(1 \leq i<j \leq n), i=1, \ldots, n, j=1, \ldots, n$

$$
\frac{y-y_{i}}{x-x_{i}}=\frac{y_{j}-y_{i}}{x_{j}-x_{i}}
$$

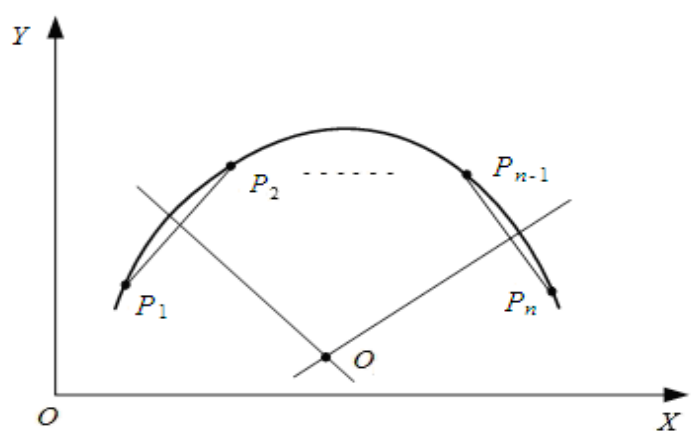

Figure 3.3. The measuring points of a woman's chest

The equation of mid perpendicular of $P_{\mathrm{i}} P_{\mathrm{j}}$ is

$$
y=-\frac{x_{j}-x_{i}}{y_{j}-y_{i}}\left(x-\frac{x_{i}+x_{j}}{2}\right)+\frac{y_{i}+y_{j}}{2}
$$

For (3.2), there are $n(n-1) / 2$ equations to form a system of linear equations (3.3). The least squares method is used to solve the system of linear equations and the result is the optimal position of the center of the circle. Suppose

$$
\begin{aligned}
& a_{i j}=\frac{x_{j}-x_{i}}{y_{j}-y_{i}}, b_{i j}=\frac{x_{j}-x_{i}}{y_{j}-y_{i}} \times \frac{x_{i}+x_{j}}{2}+\frac{y_{i}+y_{j}}{2}, \quad(1 \leq i<j \leq n)
\end{aligned}
$$

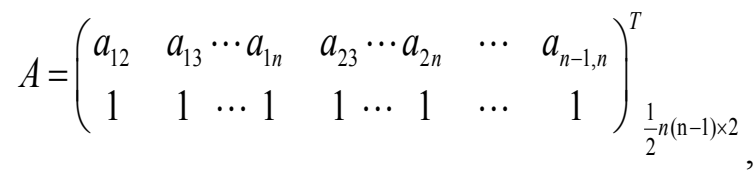

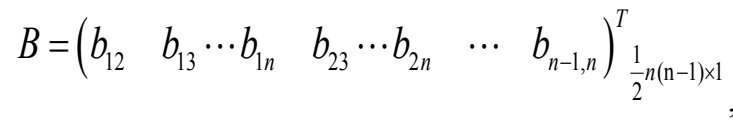

$$
X=(x, y)^{T}
$$

The system of linear equations is

$$
A X=B \text {. }
$$

The solution of least squares method is

$$
X_{L S}=\left(A^{T} A\right)^{-1} A^{T} B \text {. }
$$

So the center of circle is

$$
O\left(x_{0}, y_{0}\right)=X_{L S}^{T}
$$

Because the sphere bounding box will include all measuring points, then the optimal radius is calculated by the maximum distance from measuring points to the circle center, namely $R=\max \left\{P_{i} O\right\}, i=1, \ldots, n$. The result is illustrated in Figure 3.4.

\subsubsection{Hip}

The hip is also approximated by a sphere bounding box. The construction is similar to the method for the woman's chest. The result is illustrated in Figure 3.5.
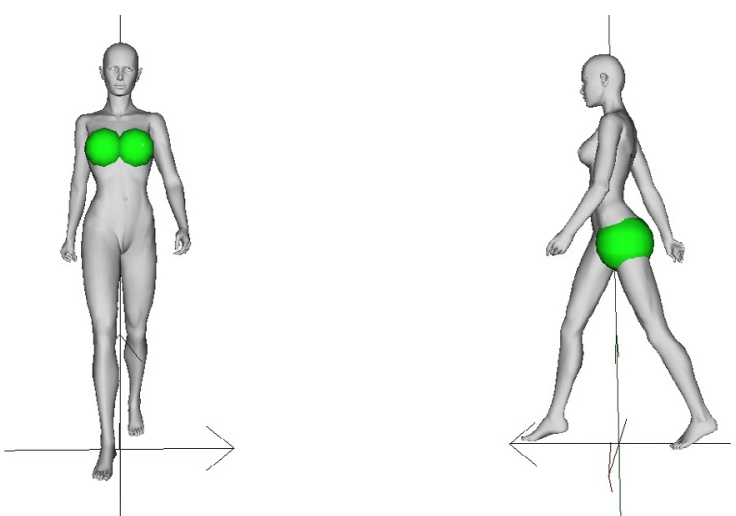

Figure 3.4. Woman's chest bounding box Figure 3.5. Hip bounding box

\subsubsection{Trunk}

The trunk is replaced by an elliptic cylinder bounding box in the simulation. A rectangle is first constructed by using the foremost point of the front and side of the torso based on the direction that the human model faces. Then a circumscribed ellipse from the rectangle is created. Finally an elliptic cylinder bounding box is achieved by combining with the height of the trunk. The result is illustrated in Figure 3.6.

\subsubsection{The overall effect}

After the construction of different kinds of bounding boxes, the overall effect approximating the human body model is illustrated in Figure 3.7. In this figure, blue parts are sphere bounding boxes, red parts are cylinder bounding boxes, green parts are elliptic cylinder bounding boxes. 


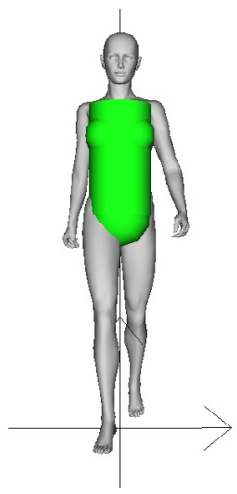

Figure 3.6. Trunk bounding box

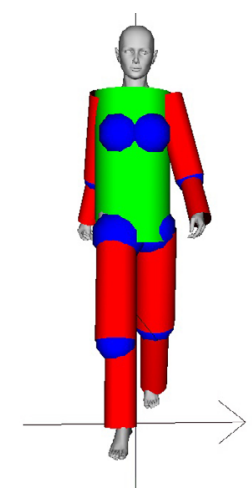

Figure 3.7. The overall effect

\subsection{Collision detection and response}

\subsubsection{Cylinder bounding box}

A cylinder bounding box is used to simulate the leg and arm. The parameters of the cylinder bounding box are a center line segment $L$ denoting the height of the cylinder bounding box (two endpoints of the line segment are $Y_{0}$ and $Y_{1}$ ) and radius $r$.

For a given cylinder bounding box $C$, it produces a plane $P_{1}$ whose normal vector direction is of line segment $L$, and the plane includes the point $Y_{1}$. We firstly do the collision detection for the vertical position. The movement path of particles $x_{0} x_{1}$ is projected to line segment $L$, where the position $x_{0}$ is the current position of cloth particle and the position $x_{1}$ is the position of cloth particle at next frame. If two projective points are both out of line segment $L$, we will not execute collision detection and response for the cloth particle. Otherwise, we do the collision detection for the horizontal position. Then, the movement path of particles $x_{0} x_{1}$ is projected to the plane $P_{1}$ as $x_{0}{ }^{\prime} x_{1}{ }^{\prime}$. Collision detection is processed by the minimum distance from the circle whose center point is $Y_{1}$ to line segment $x_{0}^{\prime} x_{1}^{\prime}$ (as illustrated by Figure 3.8). Through the vertical and horizontal collision detection, we can determine the movement feature of particles from one frame to the next frame in the clothing simulation.

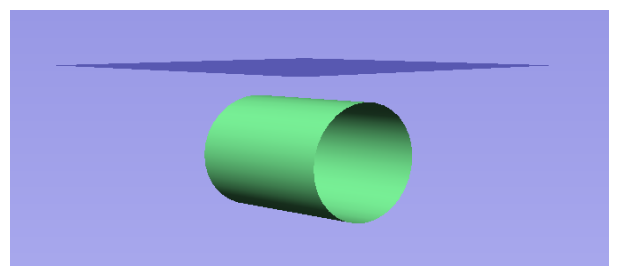

(a)

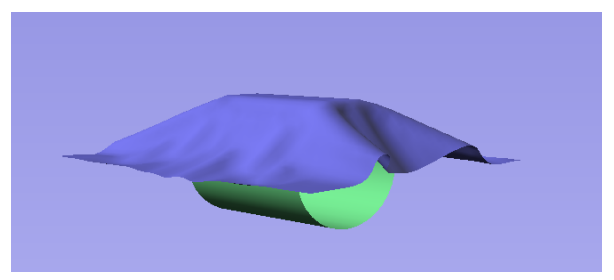

(c)

Figure 3.9. Cylinder bounding box collision with cloth

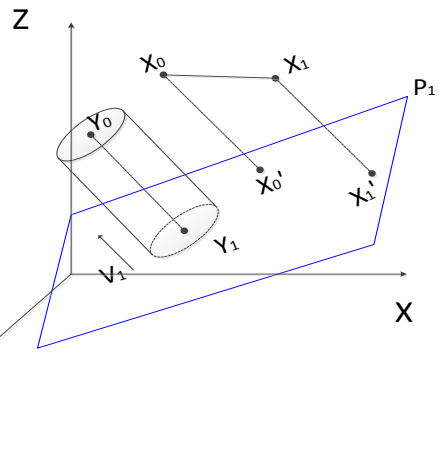

Figure 3.8. The projection of movement path of particles for cylinder bounding box

If the collision occurs, it supposes that the collision position is $x_{\mathrm{p}}{ }^{\prime}$ between the movement path $x_{0}{ }^{\prime} x_{1}^{\prime}$ and the circle. Then the actual position $x p$ is determined according to the position relation from $x_{p}{ }^{\prime}$ to $x_{0}{ }^{\prime}$ and $x_{1}{ }^{\prime}$. The collision resulting from the cylinder bounding box collision with cloth is illustrated in Figure 3.9.

\subsubsection{Elliptic cylinder bounding box}

An elliptic cylinder bounding box is used to simulate the trunk. The parameters of the elliptic cylinder bounding box are a center line segment $L$ denoting the height of elliptic cylinder bounding box (two endpoints of the line segment are $Y_{0}$ and $Y_{1}$ ) and semi-axis length $a$ and $c$ of ellipse.

For a given elliptic cylinder bounding box $C$, a plane $P_{1}$ is created whose normal vector direction is of line segment $L$, and the plane includes the point $Y_{1}$. The movement path of particles $x_{0} x_{1}$ is projected to the plane $P_{1}$ as $x_{0}{ }^{\prime} x_{1}^{\prime}$. The collision detection is processed by the minimum distance from the ellipse whose center point is $Y_{1}$ to line segment $x_{0}^{\prime} x_{1}^{\prime}$ (as illustrated by Figure 3.10).

If the collision occurs, a collision position $x_{p}^{\prime}$ is achieved between the movement path $x_{0}{ }^{\prime} x_{1}^{\prime}$ and the ellipse. Then the actual position $x p$ is determined from the relation from $x_{p}{ }^{\prime}$ to $x_{0}^{\prime}$ and $x_{1}^{\prime}$.

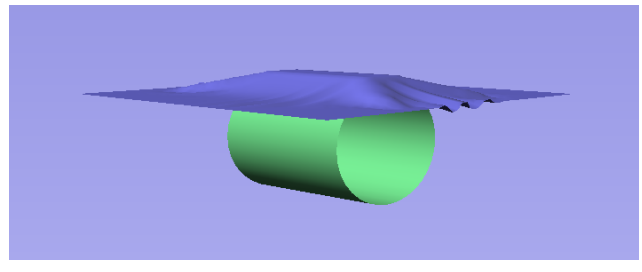

(b)

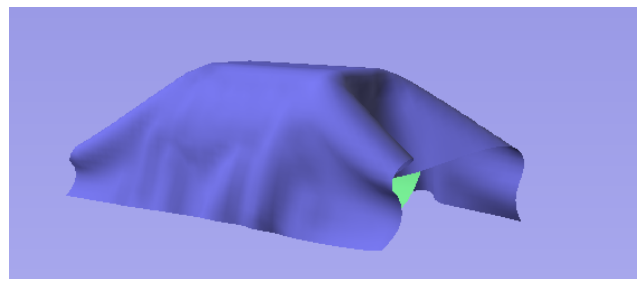

(d) 
The collision result that occurs with an elliptic cylinder bounding box collision with cloth is illustrated in Figure 3.11.

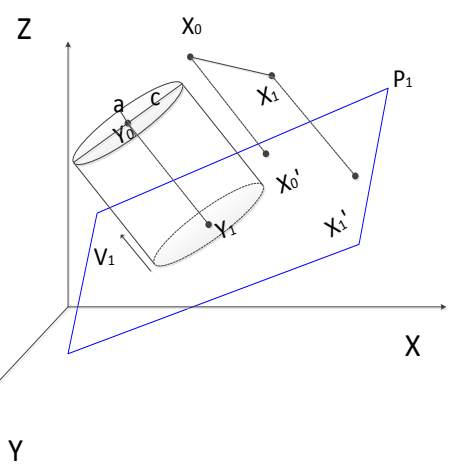

Figure 3.10. The projection of movement path of particles for elliptic cylinder bounding box

\section{Mass-spring model and numerical integration}

\section{$\underline{5.1 \text { Mass-spring model }}$}

The traditional mass-spring model [4] considers that cloth is a collection of several particles on a micro level. In order to achieve the effect of the real fabric, a spring keeps the particles connected in the relative position. Particles have no size, but have fixed quality. The method uses structure spring, shear spring and bend spring to simulate an internal force between

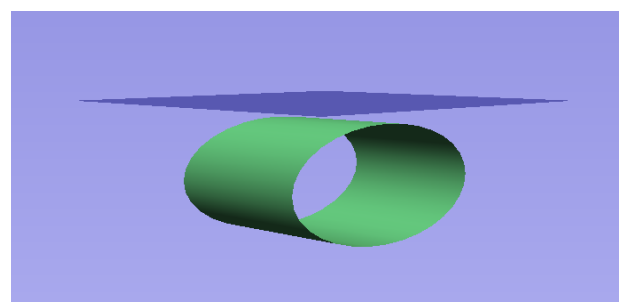

(a)

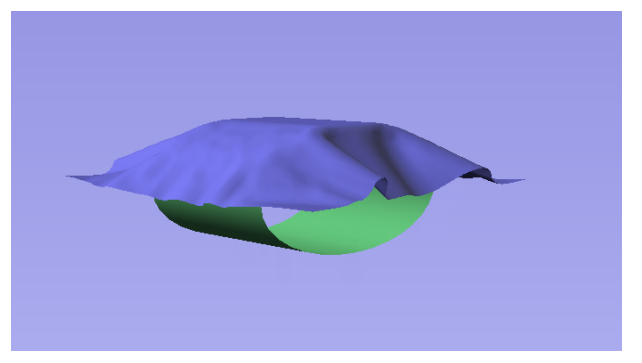

(c)

Figure 3.11. Elliptic cylinder bounding box collision with cloth
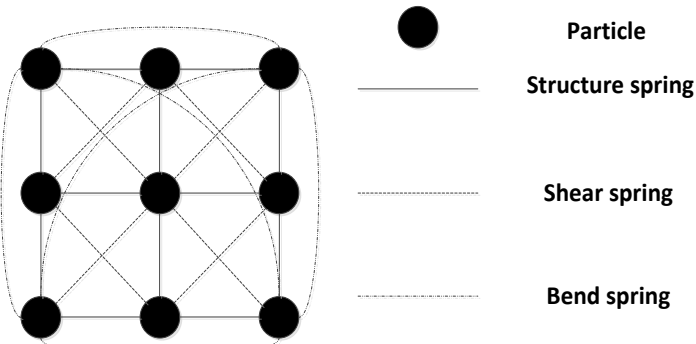

Figure 4.1. Traditional mass-spring model the particles (as illustrated by Figure 4.1). Because the cloth model is a triangular mesh, a mass-spring model based on a triangular mesh is used here. The difference of two models is that the structure spring in the triangular mesh model is combined with the shear spring and the original structure spring on the edge of the triangular face (as illustrated by Figure 4.2).

\subsection{Calculation of particles position}

The Verlet integration method ${ }^{[5]}$ is used to calculate the particles position. The method of calculation is simple, involving only the mass point position. There is no explicit solution of speed items thus the synchronization problem of speed and position does not exist. The computation process is stable and keeps the $4^{\text {th }}$ order of integration error, which is sufficient for a real-time simulation system. The Verlet integration method is an explicit method. It is derived by the Taylor expansion

$\left\{\begin{array}{l}x^{t+h}=x^{t}+h v^{t}+a h^{2} / 2+\frac{a^{\prime} h^{3}}{3 !}+O\left(h^{4}\right), \\ x^{t-h}=x^{t}-h v^{t}+a h^{2} / 2-\frac{a^{\prime} h^{3}}{3 !}+O\left(h^{4}\right) .\end{array}\right.$

So it has

$x^{t+h}=2 x^{t}-x^{t-h}+a h^{2}+O\left(h^{4}\right)$.

\section{Namely,}

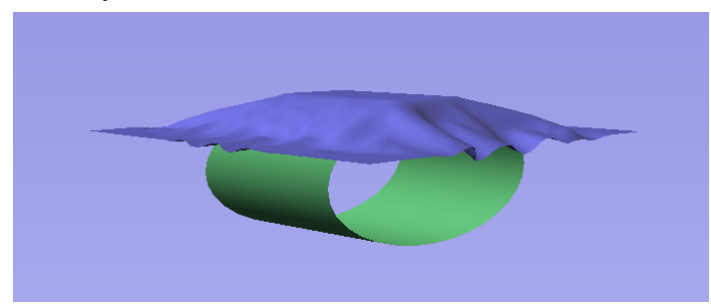

(b)

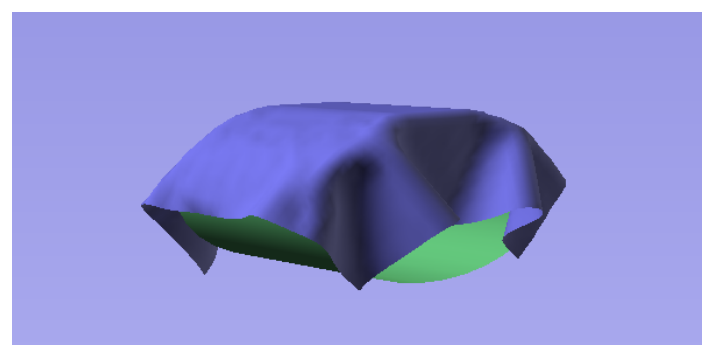

(d)

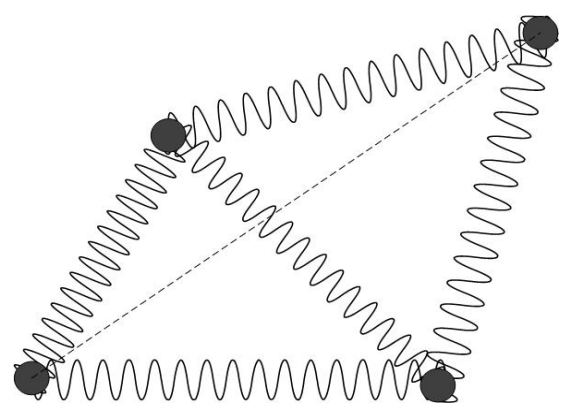

Figure 4.2. Mass-spring model based on a triangular mesh 


$$
x^{t+h}=x^{t}+\left(x^{t}-x^{t-h}\right)+a h^{2}+O\left(h^{4}\right) .
$$

where $h$ is the time step, $x t$ is the position at time $t, x r^{+} h$ is the position at time $t+h, a$ is the acceleration of particle.

\section{Experimental results and analysis}

We used the above methods to construct different bounding boxes for a human body model and built a spring-mass model for cloth to realize the garment simulation. The collision detection was performed by hybrid bounding volume hierarchy to get a realistic simulation effect. The whole simulation was implemented in $\mathrm{C}++$ and was run on computers with Core i72675QM and 4GB RAM.

\section{$\underline{6.1 \text { Simulation results of body garment }}$}

The dynamic simulation was tested on different cloth models, such as dress, jacket, skirt and trousers. Different cloth mesh models are shown in Figure 5.1 and the number of vertices and triangular facets of cloth models are shown in Table 5.1. In our clothing simulation, we add some geometric constrains for vertices on the upper part of clothing model. For example,
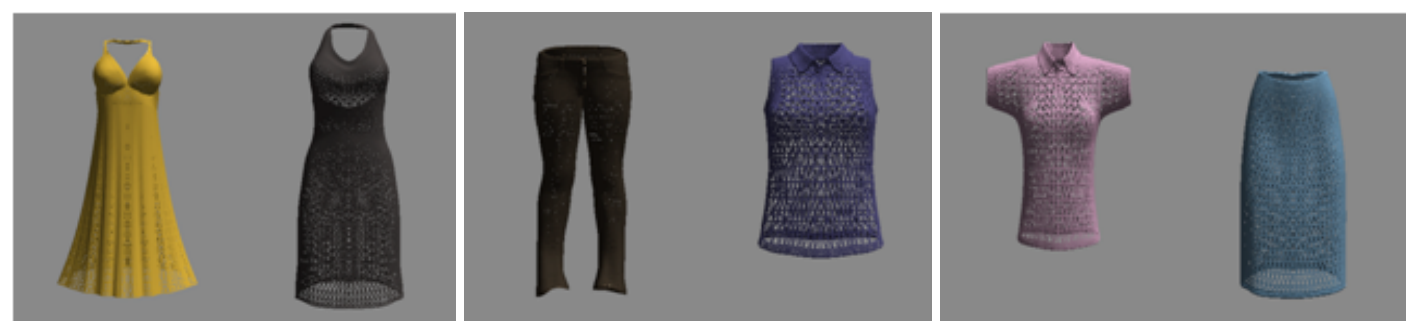

Table 5.1 Simulation Data in the upper part of the trunk and the arm, if we directly use our bounding volumes for the collision detection, the part of clothing around this region will not be close to the human body. We fix the geometric position of the upper part of clothing to the corresponding position of the human body with a constant small distance. This operation can help us reduce the collision detection for these parts of clothing and does not obviously influence the overall dynamic simulation effect. The simulation results of the dress are shown in Figure 5.2- 5.3. Figure 5.4 illustrates the results of a skirt 1 and T-shirts 1 . Figure 5.5 shows the results of a skirt 2 and T-shirts 2 .

\begin{tabular}{|c|c|c|}
\hline Cloth & $\begin{array}{c}\text { The number of } \\
\text { vertices }\end{array}$ & $\begin{array}{c}\text { The number of } \\
\text { triangular facets }\end{array}$ \\
\hline Dress 1 & 11912 & 3460 \\
\hline Dress 2 & 8853 & 2714 \\
\hline Skirt & 4612 & 1353 \\
\hline Trousers & 7158 & 2416 \\
\hline
\end{tabular}

Figure 5.1. Cloth mesh models
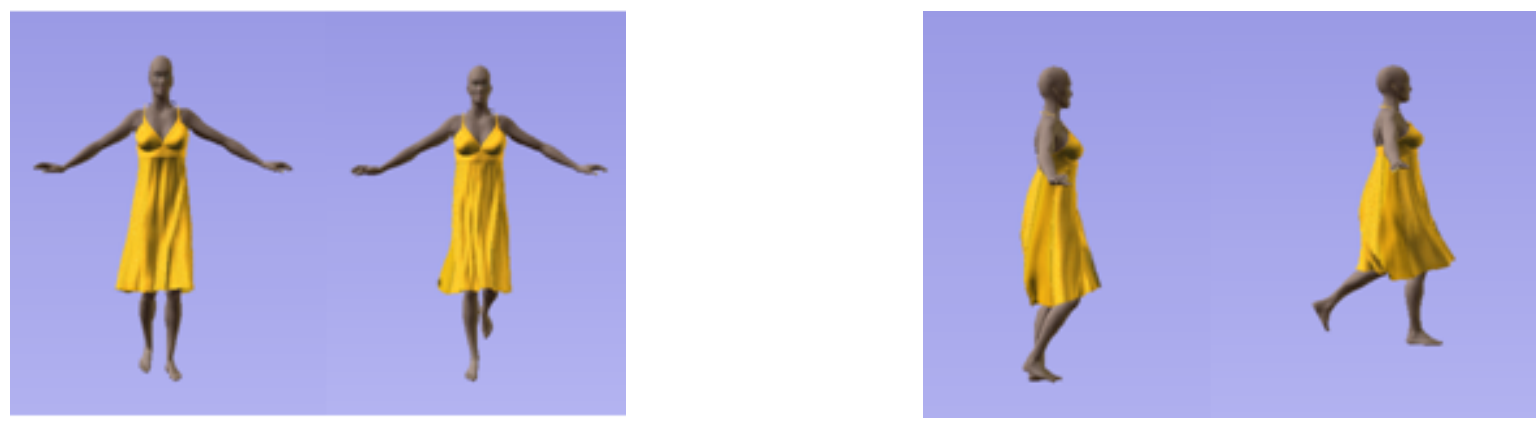

Figure 5.2. Results of dress 1
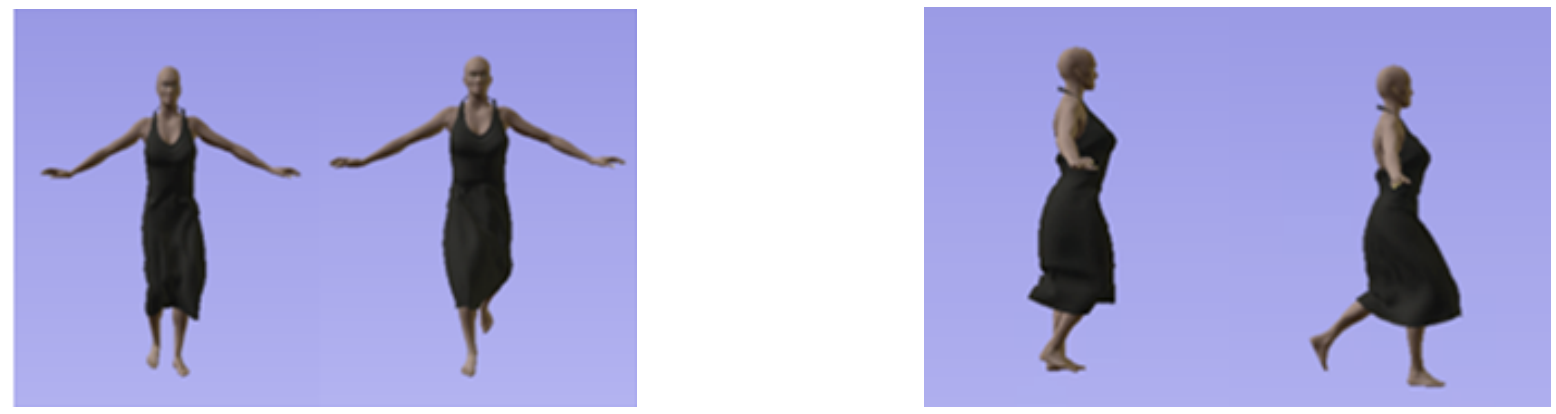

Figure 5.3. Results of dress2 


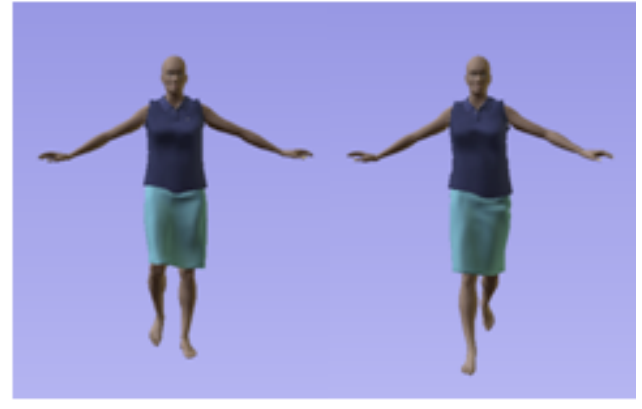

Figure 5.4. Results of skirt 1 and T-shirts 1

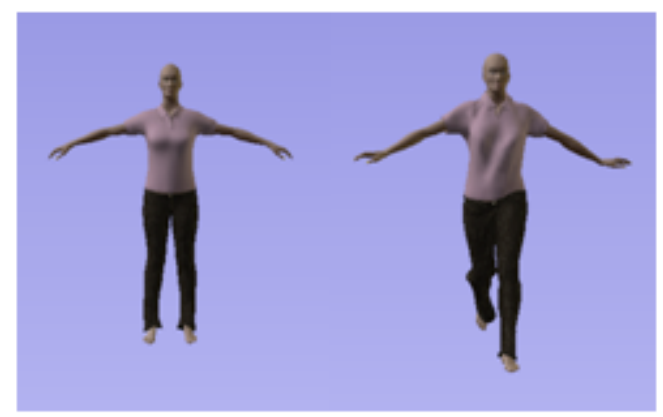

Figure 5.5. Results of skirt 2 and T-shirts 2

\section{$\underline{6.2 \text { Data Analysis }}$}

In the cloth simulation, it was found that the processing speed of each simulation frame is associated with the number of collision particles. Table 5.2 provides the time of different cloth simulation. It includes

a) The initial time of generating mass-spring model by triangular facets and the free-fall of cloth.

b) The shortest time in each frame for different cloth simulation.

c) The maximum time in each frame for different cloth simulation.

At the free-fall state of the cloth models, we recorded the number of collision particles and found it rises at the beginning of cloth simulation. After it attains the peak value, the number of collision particles tends to be stable (as illustrated by Figure 5.6).

We also compared our method with hybrid bounding volume hierarchy to the methods based on sphere bounding box and ellipsoid bounding box. The collision detection time is illustrated in Table 5.3. The advantage of our method is that it can improve the collision detection, because we construct the simple volume box to approximate the part of the body model, the collision detection is speeded up, which is shown in Table 5.3. The connection of cloth shaping and the body can also be realized through the response of the improved collision detection.

\section{Conclusion and future work}

In this paper, a method of using MCASG graph and K-means clustering algorithm to process the segmentation was
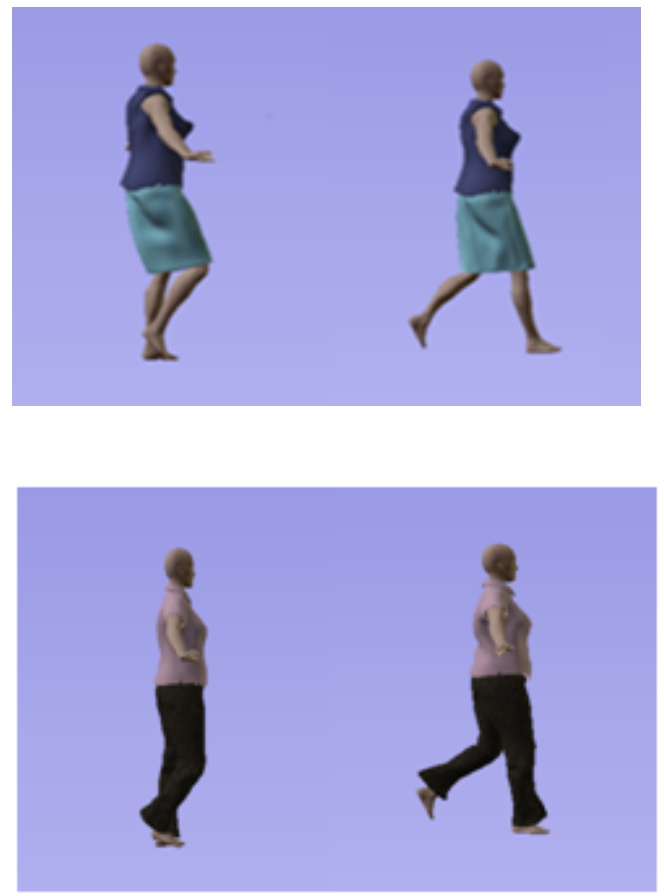

Table 5.2 Time of cloth simulation

\begin{tabular}{|c|c|c|c|}
\hline Cloth & $\begin{array}{c}\text { Initial } \\
\text { time }\end{array}$ & $\begin{array}{c}\text { Shortest time } \\
\text { of each frame }\end{array}$ & $\begin{array}{c}\text { Maximum time } \\
\text { of each frame }\end{array}$ \\
\hline Dress 1 & $1.53 \mathrm{~s}$ & $83 \mathrm{~ms}$ & $166 \mathrm{~ms}$ \\
\hline Dress 2 & $1.12 \mathrm{~s}$ & $58 \mathrm{~ms}$ & $111 \mathrm{~ms}$ \\
\hline Skirt & $0.60 \mathrm{~s}$ & $32 \mathrm{~ms}$ & $62 \mathrm{~ms}$ \\
\hline Trousers & $1.07 \mathrm{~s}$ & $43 \mathrm{~ms}$ & $83 \mathrm{~ms}$ \\
\hline
\end{tabular}

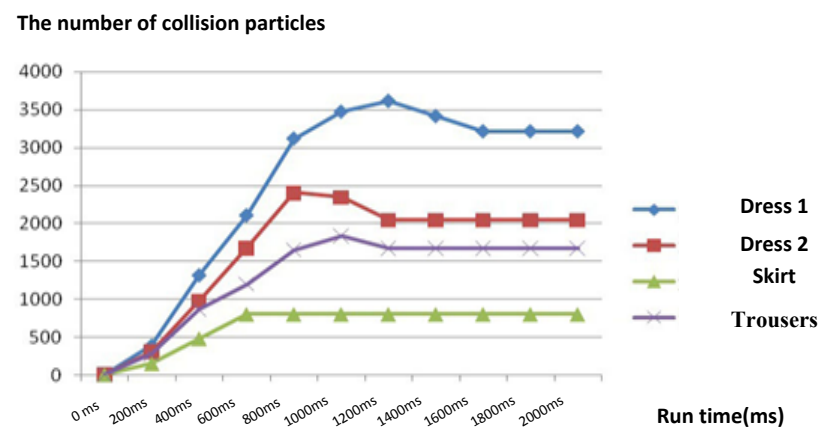

Figure 5.6: Change chart of the number of collision particles

Table 5.3 Running time of collision

\begin{tabular}{|c|c|c|c|}
\hline Cloth & $\begin{array}{c}\text { Ellipsoid } \\
\text { bounding } \\
\text { box }\end{array}$ & $\begin{array}{c}\text { Sphere } \\
\text { bounding } \\
\text { box }\end{array}$ & Our method \\
\hline Dress 1 & $152 \mathrm{~ms}$ & $212 \mathrm{~ms}$ & $83.3 \mathrm{~ms}$ \\
\hline Dress 2 & $168 \mathrm{~ms}$ & $217 \mathrm{~ms}$ & $84.6 \mathrm{~ms}$ \\
\hline Skirt & $128 \mathrm{~ms}$ & $182 \mathrm{~ms}$ & $43.5 \mathrm{~ms}$ \\
\hline Trousers & $103 \mathrm{~ms}$ & $148 \mathrm{~ms}$ & $32.3 \mathrm{~ms}$ \\
\hline
\end{tabular}


presented. Different bounding boxes approximating the units of the body model with the shape features were described. Then it was shown how a mass-spring model based on a triangular mesh for processing collision detection and response was introduced. This method could improve the efficiency of collision detection to realize fast and realistic clothing simulation.

In our current simulation, we mainly realize the clothing shaping through the collision detection for the bottom areas of the clothing model. For the upper part of the clothing model, it is geometrically constrained which keeps the part of clothing close to the human body model. How to achieve more realistic simulation for the upper part of clothing and some tight cloth will be our future work.

\section{Acknowledgements}

This research was supported by Natural Science Foundation of China under Grant No. 11671009, Scientific Research Foundation of Ministry of Education of China under Grant No. [2009]1590, Zhejiang Provincial Natural Science Foundation of China under Grant No. LY14A010032, Zhejiang Province Key Science and Technology Innovation Team Project (2013TD18) and Project of 521 Excellent Talent of Zhejiang Sci-Tech University.

\section{References}

[1] Liu T, Bargteil A W, O'Brien J F, et al. Fast simulation of mass-spring systems[J]. ACM Transactions on Graphics (TOG), 2013, 32(6), 214.

[2] Wong $T H$, Leach G, Zambetta F. Modelling Bending Behaviour in Cloth Simulation Using Hysteresis[C]. Computer Graphics Forum. 2013, 32(8), 183-194.

[3] Kavan L, Gerszewski D, Bargteil A W, et al. Physicsinspired upsampling for cloth simulation in games[C]. ACM Transactions on Graphics (TOG). ACM, 2011, 30(4), 93.

[4] Provot $X$. Deformation constraints in a mass-spring model to describe rigid cloth behaviour [C]. Graphics interface. Canadian Information Processing Society, 1995, 147-147.

[5] Jakobsen T. Advanced character physics[C]. Game Developers Conference, 2001, 383-401.

[6] Miguel E, Tamstorf R, Bradley $D$, et al. Modeling and estimation of internal friction in cloth[J]. ACM Transactions on Graphics (TOG), 2013, 32(6), 212.

[7] Eberhardt B, Weber A, Strasser W. A fast, flexible, particlesystem model for cloth draping[J]. Computer Graphics and Applications, IEEE, 1996, 16(5), 52-59.
[8] Volino P, Magnenat-Thalmann N. Comparing efficiency of integration methods for cloth simulation[C]. Computer graphics international 2001. Proceedings. IEEE, 2001, 265-272.

[9] Volino $P$, Magnenat-Thalmann $N$. Implicit midpoint integration and adaptive damping for efficient cloth simulation[J]. Computer Animation and Virtual Worlds, 2005, 16(3-4), 163-175.

[10] Baraff D, Witkin A. Large steps in cloth simulation[C]. Computer Graphics (SIGGRAPH' 98), 1998, 43-54.

[11] Choi K J, Ko H S. Stable but responsive cloth[C]. ACM SIGGRAPH 2005 Courses. ACM, 2005, 1.

[12] Eberhardt B, Etzmuß O, Hauth M. Implicit-explicit schemes for fast animation with particle systems[M]. Springer Vienna, 2000.

[13] Bridson R, Marino S, Fedkiw R. Simulation of clothing with folds and wrinkles[C]. Proceedings of ACM SIGGRAPH/ Eurographics symposium on Computer animation. Eurographics Association, 2003, 28-36.

[14] Li Z, Li L, Zou F. 3D foot and shoe matching based on OBB and $A A B B[J]$. International Journal of Clothing Sciences \& stechnology, 2013, 25(5), 389-399.

[15] Feng W, Yu Y, Kim B. A deformation transformer for realtime cloth animation[J]. Acm Transactions on Graphics, 2010, 29(4), 157-166.

[16] Bischoff S, Kobbelt L. Ellipsoid decomposition of $3 D$-models[C]. Proceedings of International Symposium on 3D Data Processing, Visualization and Transmission, 2002, 480-488.

[17]Bergen G. Efficient collision detection of complex deformable models using $A A B B$ trees[J]. Journal of Graphics Tools, 1997, 2(4), 1-13.

[18] Hutter M, Fuhrmann A. Optimized continuous collision detection for deformable triangle meshes[C]. In Proc. WSCG '07, 2007, 25-32.

[19] Chang J W, Wang W, Kim M S. Efficient collision detection using a dual OBB-sphere bounding volume hierarchy[J]. Computer-Aided Design, 2010, 42(1), 50-57.

[20] Jagannathan A, Miller $E$ L. Three-dimensional surface mesh segmentation using curvedness-based region growing approach[J]. IEEE Transactions on Pattern Analysis and Machine Intelligence, 2007, 29(12), 21952204.

[21] Hartigan J A, Wong M A. Algorithm AS 136: A k-means clustering algorithm[J]. Applied Statistics, 1979: 100-108.

[22] Dyn N, Hormann K, Kim S J, et al. Optimizing $3 D$ triangulations using discrete curvature analysis[J]. Mathematical methods for curves and surfaces, 2001, 135-146.

[23] Alexa M, Behr J, Cohen-Or D, et al. Computing and rendering point set surfaces[J]. IEEE Transactions on Visualization and Computer Graphics, 2003, 9(1), 3-15. 